\title{
The Kinetics and Formation of Small Carbon Clusters in an Argon Matrix
}

\author{
Jan Szczepanski, Robert Pellow, and Martin Vala \\ Department of Chemistry, University of Florida, Gainesville, Florida, USA
}

Z. Naturforsch. 47a, 595-604 (1992); received November 25, 1991

\begin{abstract}
Pure carbon clusters, formed by Nd/YAG laser ablation of graphite, have been trapped in an argon matrix at $12 \mathrm{~K}$. The temperature dependence of the prominent infrared absorption bands attributable to $C_{3}, C_{5}, C_{6}$, and $C_{9}$ clusters has been investigated between $12 \mathrm{~K}$ and $38 \mathrm{~K}$. The former two are observed to decay and the latter two to grow with increased matrix annealing. A new kinetic model based on solid state diffusion theory and involving thermally activated diffusion and aggregation is introduced. All possible pairwise interactions of the linear carbon clusters from $C_{1}$ to $C_{10}$ are included in the model. The coupled differential equations are solved numerically and provide a reasonable fit to the experimental temperature profiles of the known clusters. Predictions emerge from the fit for the unknown matrix infrared frequencies for the $C_{7}$ cluster. On the basis of the predicted concentration invariance with temperature for $C_{7}$, the infrared bands at $2128 \mathrm{~cm}^{-1}$ and $1894 \mathrm{~cm}^{-1}$ are ascribed to this linear cluster. This attribution supports the recent assignment of the gaseous $2138 \mathrm{~cm}^{-1}$ band to linear $C_{7}$.
\end{abstract}

\section{Introduction}

Carbon clusters have attracted much attention recently because of their probable importance in certain aspects of astrophysics, soot formation and flame chemistry ${ }^{1}$. Carbon vaporized from graphite and trapped in a cryogenic rare gas matrix displays numerous infrared bands in the carbon-carbon stretching region indicating the presence of many absorbing species, i.e. different carbon clusters ${ }^{2-5}$. It has been known for some time ${ }^{2}$ that annealing the matrix can cause the relative intensities of bands either to decrease, to increase, to disappear or to appear for the first time. While growth (or decay) patterns of some bands have been reported in the literature ${ }^{2-5}$, no systematic study of their behavior with temperature has been reported. For such a study to succeed, the infrared bands must be conclusively attributed to specific clusters. Previously this would not have been possible, but now, through the combined efforts of matrix isolation and vapor phase investigation in concert with theoretical studies, a number of IR assignments are becoming firmed up. Recent developments are briefly reviewed here. In the present paper we report on the growth and decay of the major small $(n \leq 9)$ linear carbon clusters as a function of matrix annealing. In addition, a new kinetic model is intro-

Reprint requests to Prof. M. Vala, Department of Chemistry, University of Florida, Gainesville, FL 32611-2046/USA duced which accounts reasonably well for the observed growth and decay patterns.

Spectroscopic kinetic studies of the formation of small clusters have not been numerous ${ }^{6-11}$. Yamada, Usui, and Takagi ${ }^{6}$ and Castleman and Keesee ${ }^{7}$ investigated the kinetics and formation of metal clusters during expansion through a nozzle. In each case a model built on classical nucleation theory was utilized to extract information on cluster formation. Bernholc and Phillips simulated gas-phase carbon cluster aggregation for neutral and charged clusters ${ }^{8}$. The Smoluchowski equations were solved using electronic structure calculations to obtain the needed kinetic parameters. Good agreement with experimental data was achieved. Ozin and Huber used a simplified version of the Smoluchowski diffusion-controlled kinetic theory to explain their photoaggregation results for small Ag clusters in argon matrices ${ }^{9}$. Moskovits and Hulse introduced a quenched reaction model $^{10}$ in which successive additions of monomer (or ligand) to the growing cluster (complex) were considered. Applied to the formation of $\mathrm{Ni}_{x}(\mathrm{CO})_{y}$ complex clusters, the model was shown to give an acceptable fit to the data. Nishiya et al. extended the quenched reaction model to include new channels for cluster formation (addition of dimers and trimers) in their IR study of ammonia clusters ${ }^{11}$. In the present work we adopt the full Smoluchowski diffusion-controlled kinetic model $^{12}$ and allow for the formation of linear carbon 


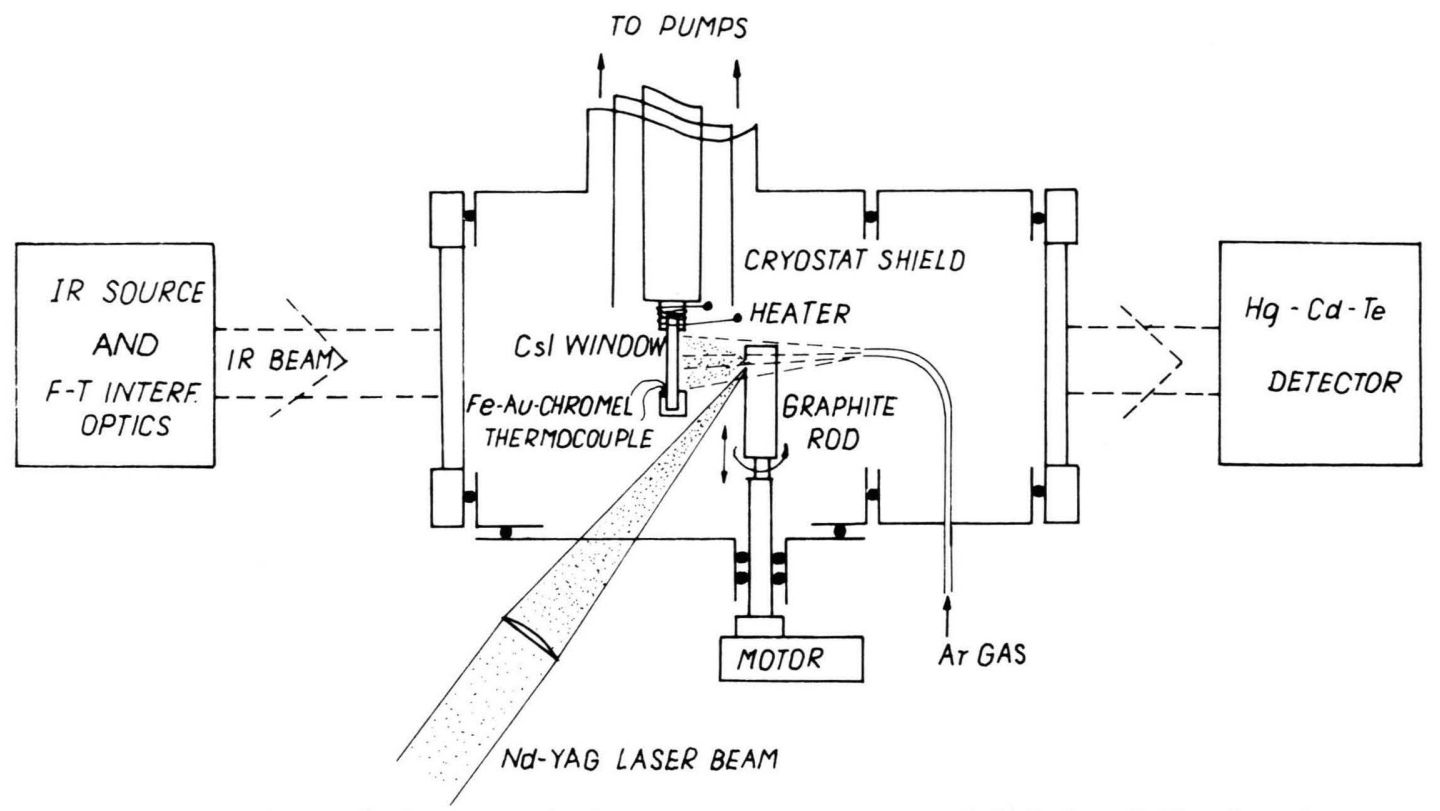

Fig. 1. Experimental setup for laser vaporization of graphite rod and matrix isolation of ablated species.

clusters via all possible routes (i. e., addition of monomers, dimers, trimers, etc.).

The paper is arranged as follows. The experimental details are described in Section II. A brief review of the current state of knowledge of the IR assignments of the small carbon clusters is given in Section III. In Section IV, the annealing results are presented (IVA), followed by some possible limitations on aggregation reactions (IVB) and then a description of the carbon cluster reaction model (IVC). Section IVD gives details on the computational approach used and, finally, the results are discussed in Section V.

\section{Experimental}

The apparatus and procedures used have been described previously ${ }^{13}$, so only a brief description is given here. The carbon clusters were produced by laser ablation of graphite using a slightly focussed Q-switched Nd/YAG laser (Spectra Physics, DCR-11, $532 \mathrm{~nm}, 1.0 \mathrm{~mJ} /$ pulse, $10 \mathrm{~Hz}$ rep. rate). A schematic of the vaporization/deposition cell is shown in Figure 1. The ablated graphite products were trapped with argon $(99.995 \%$ pure, Matheson) at $12 \mathrm{~K}$ on a CsI window mounted on the cold finger of a closed-cycle helium cryostat (Displex DE 202, APD). Under similar conditions, both $\mathrm{ESR}^{14}$ and previous $\mathrm{IR}^{4,13}$ mea- surements have shown that large quantities of $C_{3}, C_{5}$, and $C_{6}$ clusters are produced and trapped.

Infrared spectra were obtained on a Nicolet FTIR spectrometer using 200 scans with $1 \mathrm{~cm}^{-1}$ resolution. After a scan at $12 \mathrm{~K}$, the matrix was annealed for $10 \mathrm{~min}$ at an elevated temperature (from 23 to $38 \mathrm{~K}$ ), then returned to $12 \mathrm{~K}$ where another spectrum was collected. This procedure was then repeated at a higher annealing temperature.

\section{Review of Carbon Cluster IR Assignments}

Since one of the major goals of this work is to study the aggregation of carbon clusters in an argon matrix by investigating the temperature dependence of the infrared bands of the clusters, it is imperative that the specific IR bands followed be well-assigned. In this section we give a brief review of the present status of the IR band assignments of the small $(n \leq 9)$ carbon clusters, with emphasis on matrix studies. An excellent review through April 1989 has been provided by Weltner and Van Zee ${ }^{1}$, but there have been significant advances since then.

\section{A) $C_{3}$}

Probably the best known of the small clusters, $C_{3}$ was studied in matrices using isotopic substitution by 
Weltner and coworkers ${ }^{2,15,16}$ who proved conclusively that the $2039 \mathrm{~cm}^{-1}$ (Ar matrix) band originated from linear $C_{3}$.

B) $C_{4}$

Using isotopically labelled acetylene, diacetylene and butadiene, Shen and Graham showed ${ }^{17}$ that the $1544 \mathrm{~cm}^{-1}$ IR band originates from linear $C_{4}$. Laser ablation studies have not been successful in producing intense enough bands in this region to discern isotopomeric structure ${ }^{18}$.

C) $C_{5}$

An early assignment of the $2164 \mathrm{~cm}^{-1}$ Ar matrix band to $C_{4}$ was proven to be erroneous, when a laservaporized, matrix-deposited $1: 1$ mixtrue of ${ }^{12} \mathrm{C}:{ }^{13} \mathrm{C}$ was shown ${ }^{18}$ to exhibit twenty isotopomeric peaks. For linear $C_{4}$ only 10 peaks are predicted ${ }^{19}$. For $C_{5}$, the most reasonable structures and the expected number of isotopomers for each (in parentheses) are trigonal bipyramidal (12), planar cyclic (8) and linear (20). A normal coordinate analysis of the linear form provided strong support ${ }^{18}$ for the assignment of the $2164 \mathrm{~cm}^{-1}$ band to linear $C_{5}$. Soon thereafter, studies of vapor phase $C_{5}$ confirmed this assignment ${ }^{20}$. At about the same time, $C_{5}$ was discovered ${ }^{21}$ in the cool shell of a carbon star by the rotational analysis of the infrared band system at $2169 \mathrm{~cm}^{-1}$.

D) $C_{6}$

A prominent band at $1952 \mathrm{~cm}^{-1}$ has been shown ${ }^{4}$ to exhibit 35 isotopomeric peaks. Thirty-six are expected for linear $C_{6}$. Normal coordinate analysis on near-linear $C_{6}$ provided strong support for the assignment of this band to cumulenic $C_{6}$; of the 36 expected peaks two were found to be overlapped. This assignment has been confirmed by Martin, Francois, and Gijbels using MP2/6-31G* level $a b$ initio theoretical calculations $^{23}$.

\section{E) $\boldsymbol{C}_{7}$}

No matrix studies have been reported which provide conclusive evidence for the IR assignment of this species. A very recent vapor phase-study by Saykally and co-workers ${ }^{24}$ shows that a band at $2138 \mathrm{~cm}^{-1}$ results from the linear cumulenic $C_{7}$ cluster. In this paper we provide evidence that the
$2128 \mathrm{~cm}^{-1}$ and $1894 \mathrm{~cm}^{-1}$ matrix bands are to $C_{7}$ (vide infra).

\section{F) $C_{9}$}

The temperature-sensitive band at $1998 \mathrm{~cm}^{-1}$ had been assigned tentatively ${ }^{4}$ to $C_{8}$. This assignment relied on band density and isotopomeric peak intensity arguments. For linear $C_{8}, 136$ isotopomeric peaks are expected ${ }^{19}$ in a span of $\sim 78 \mathrm{~cm}^{-1}$. Over one hundred isotopomeric bands built on the $1998 \mathrm{~cm}^{-1}$ band were observed, but considerable band overlapping is present. Although this observation clearly rules out in earlier $C_{6}$ assignment ${ }^{2}$, it is consistent with attribution to either a $C_{8}$ or higher carbon cluster. This assignment to $C_{8}$ has been questioned on theoretical grounds by Martin and co-workers ${ }^{23}$ who favor a $C_{9}$ assignment. Heath and Saykally have observed a gasphase band at $2014 \mathrm{~cm}^{-1}$ which they attribute ${ }^{25 a}$ to linear $C_{9}$. They further suggest ${ }^{25 \mathrm{~b}}$ on the basis of a comparison of gas-phase frequencies of $C_{3}, C_{5}, C_{7}$, and $C_{9}$ with certain matrix frequencies that there is a linear gas-to-matrix frequency shift which increases with cluster size and that the $1998 \mathrm{~cm}^{-1}$ matrix band should be attributed to $C_{9}$. In a recently completed isotopic matrix study ${ }^{26}$ in the IR, we have confirmed that the $1998 \mathrm{~cm}^{-1}$ band is due to $C_{9}$.

\section{Results}

\section{A) Annealing Studies}

The temperature dependence $(12 \mathrm{~K}-36 \mathrm{~K})$ of the infrared spectra of the carbon clusters produced via laser ablation is shown in Figure 2. To emphasize the changes produced upon annealing, the difference spectrum $(30 \mathrm{~K}$ spectrum minus $12 \mathrm{~K}$ spectrum) is shown in Figure 3. The prominent negative bands belong to $C_{3}\left(2039 \mathrm{~cm}^{-1}\right)$ and $C_{5}\left(2164 \mathrm{~cm}^{-1}\right)$, indicating their diminution upon annealing. The important positive difference bands belong to $C_{6}\left(1952 \mathrm{~cm}^{-1}\right)$ and $C_{9}$ $\left(1998 \mathrm{~cm}^{-1}\right)$, indicating that they increase upon warming the matrix. While this difference spectrum indicates the overall result of annealing, it does not indicate the detailed changes which occur with each temperature increment. This is provided in Figure 4, where the important IR peak heights are plotted as a function of annealing temperature. $C_{5}\left(2164 \mathrm{~cm}^{-1}\right)$ increases slightly up to $23 \mathrm{~K}$ and then decreases, more or less in parallel with $C_{3}\left(2039 \mathrm{~cm}^{-1}\right) . \quad C_{6}$ 


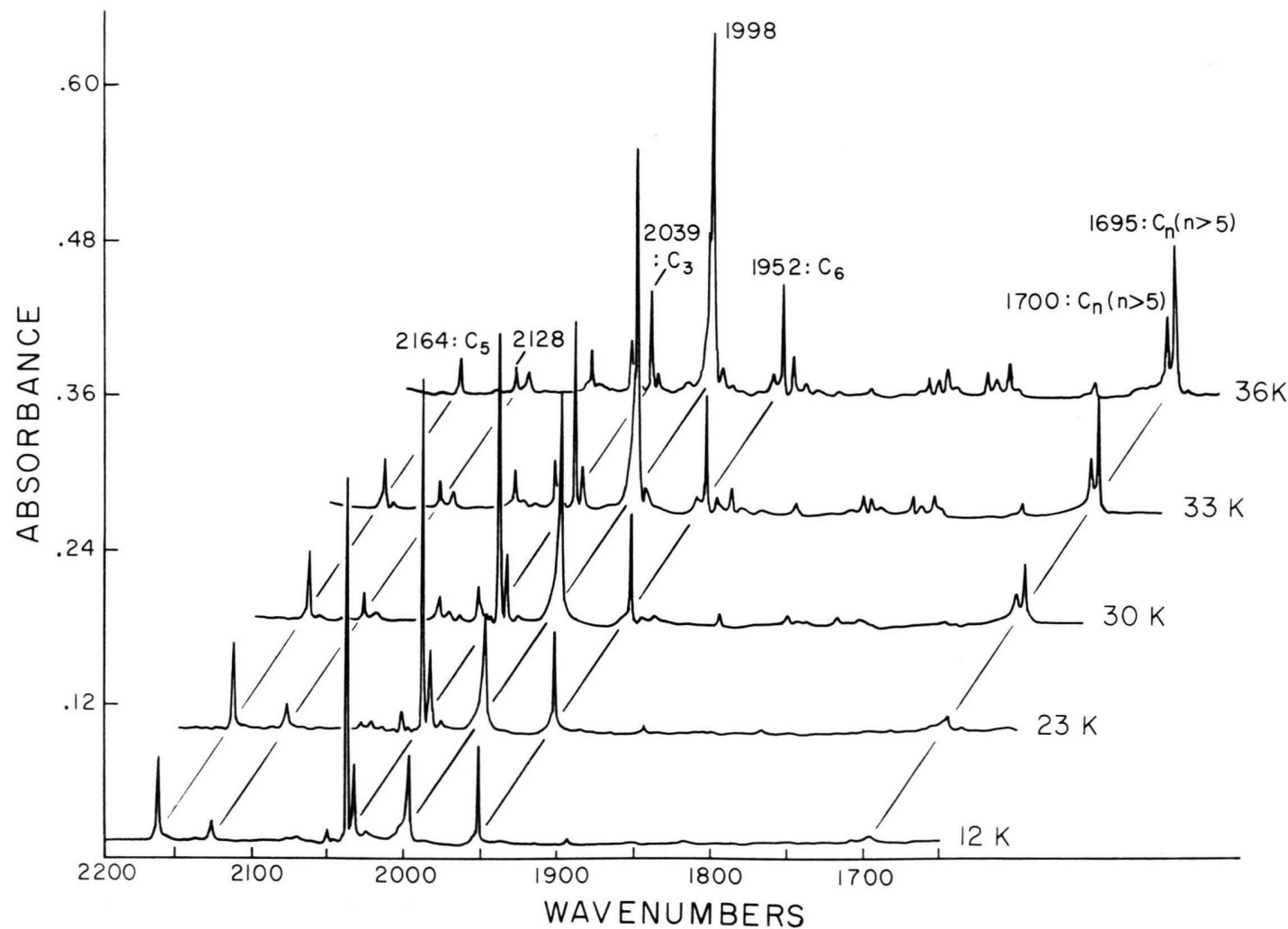

Fig. 2. Temperature dependence of the infrared spectra of carbon clusters matrix-isolated in argon. All spectra were run at $12 \mathrm{~K}$; temperatures denote the annealing temperatures.

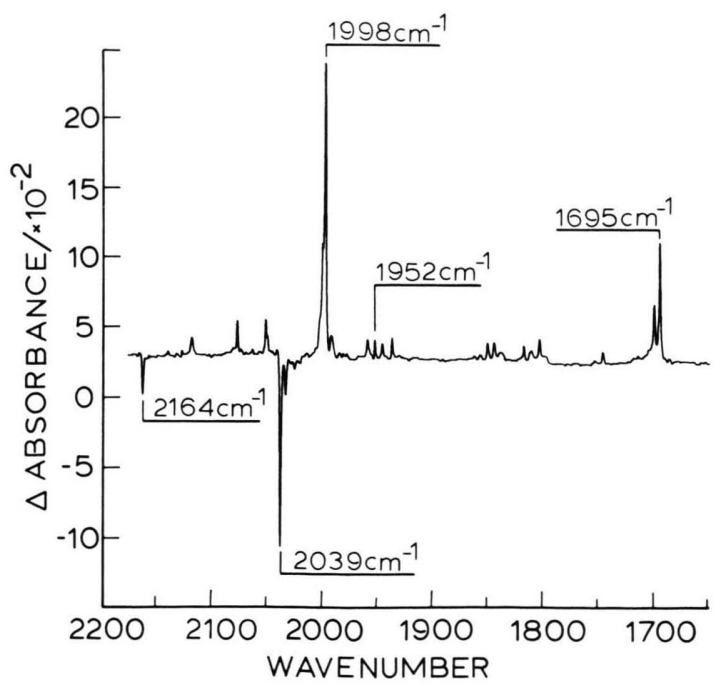

Fig. 3. IR difference spectrum (Annealed-to-30 K spectrum minus nonannealed $12 \mathrm{~K}$ one) in an argon marix.

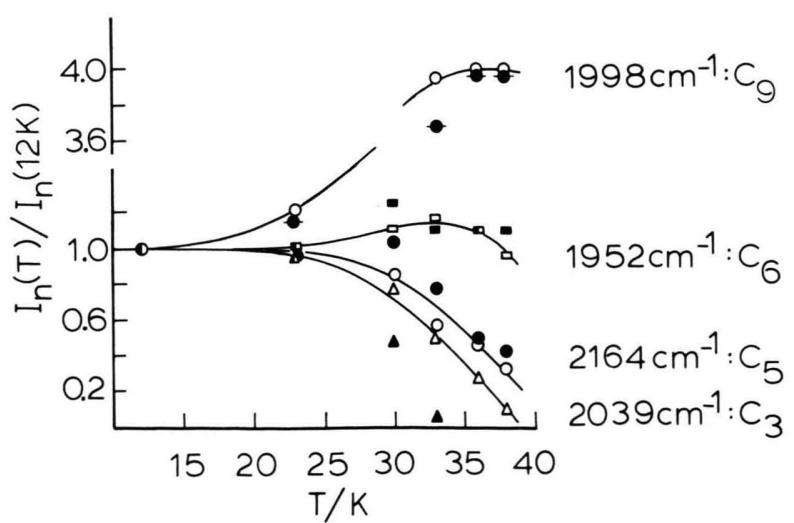

Fig. 4. Temperature vs. IR band peak intensities for specific carbon clusters in an argon matrix. The solid lines are drawn through the (open) experimental data points; the calculated points at the same temperatures are filled (black). 
$\left(1952 \mathrm{~cm}^{-1}\right)$ increases up to $\sim 33 \mathrm{~K}$ and then declines. $C_{9}\left(1998 \mathrm{~cm}^{-1}\right)$ increases markedly (factor of $\sim 4$ greater than $C_{6}$ ) up to $33 \mathrm{~K}$ and declines thereafter.

Changes in IR band intensities upon matrix annealing have been observed by previous workers. Thompson, DeKock and Weltner (TDW) suggested ${ }^{2}$ that as a result of annealing the $C_{3}$ cluster dimerized to form $C_{6}$. In our investigation, warming to $33 \mathrm{~K}$ caused the $2039 \mathrm{~cm}^{-1} C_{3}$ peak to decrease to one half its $12 \mathrm{~K}$ intensity, while the $C_{6}$ peak grew (at $33 \mathrm{~K}$ ) to only 1.2 times its $12 \mathrm{~K}$ intensity and at higher temperatures decreased. Based on these observations, it seems clear that the mechanism for the production of $C_{6}$ is more complicated than the simple dimerization of two $C_{3}$ clusters. Indeed, given the temperature-dependent behavior for the four known neutral carbon clusters shown in Fig. 4, any realistic model must be able to account for the growth and/or decay of all potentially matrix-mobile species. Such an attempt is made here. Certain limitations on specific clusters' ground state reactivity need to be recognized first, however.

\section{B) Limitations on Possible Aggregation Reactions}

The specific limitations which need to be considered are 1) decreased diffusional capability as cluster size increases and 2) conservation of spin between reactant and product clusters. In general, the heats of formation of all linear carbon clusters formed from smaller fragments are expected to be greater than $120 \mathrm{kcal} /$ mol and exothermic ${ }^{27}$. The even linear clusters $(n=4$, $6,8,10$ ) are expected ${ }^{27}$ (and are observed ${ }^{14,28}$ ) to have ${ }^{3} \Sigma_{\mathrm{g}}^{-}$ground states, while the odd clusters are predicted ${ }^{27}$ to have ${ }^{1} \Sigma_{\mathrm{g}}^{+}$ground states. Thus, spinconservation considerations ${ }^{2}$ forbid reactions such as

$$
\begin{aligned}
& 2 C_{2}\left({ }^{1} \Sigma_{\mathrm{g}}^{+}\right) \rightarrow C_{4}\left({ }^{3} \Sigma_{\mathrm{g}}^{-}\right) \\
& 2 C_{2 n-1}\left({ }^{1} \Sigma_{\mathrm{g}}^{+}\right) \rightarrow C_{2(2 n-1)}\left({ }^{3} \Sigma_{\mathrm{g}}^{-}\right), \quad n>1, \\
& C_{n}+C_{n+1} \rightarrow C_{2 n+1}, \quad n>2
\end{aligned}
$$

for all species in their ground electronic states. It may be possible, however, to form the above product clusters via excited product states, the energy provided by the exothermicity of the cluster aggregation. For example, linear $C_{6}$ could be formed in the following way $C_{3}\left({ }^{1} \Sigma_{\mathrm{g}}^{+}\right)+C_{3}\left({ }^{1} \Sigma_{\mathrm{g}}^{+}\right) \rightarrow C_{6}\left({ }^{1} \Sigma_{\mathrm{g}}^{+}\right)$, excited state.
Or via other reactant fragments utilizing only ground electronic states

$$
\begin{aligned}
& C\left({ }^{3} P_{\mathrm{g}}\right)+C_{5}\left({ }^{1} \Sigma_{\mathrm{g}}^{+}\right) \rightarrow C_{6}\left({ }^{3} \Sigma_{\mathrm{g}}^{-}\right), \text {ground state, } \\
& C_{2}\left({ }^{1} \Sigma_{\mathrm{g}}^{+}\right)+C_{4}\left({ }^{3} \Sigma_{\mathrm{g}}^{-}\right) \rightarrow C_{6}\left({ }^{3} \Sigma_{\mathrm{g}}^{-}\right), \text {ground state. }
\end{aligned}
$$

\section{C) Carbon Cluster Reaction Model}

From the above description of the annealing behavior of the known carbon clusters, it is apparent that a simple model, such as one allowing for only carbon atom diffusion in the matrix, would be inadequate. Indeed, it appears that reaction in the matrix occurs for even the largest clusters known (i. e., $C_{9}$ ). We have therefore set up a kinetic model which allows for reaction between all pairwise combinations of cluster species leading to products up through $C_{10}$. The reaction scheme is diagrammed in Figure 5. There it can be seen, for example, that $C_{6}$ may be formed via the reaction of $C+C_{5}, C_{2}+C_{4}$, or $C_{3}+C_{3}$, and it may be consumed by forming $C_{7}$ (with $C$ ), $C_{8}$ (with $C_{2}$ ), and $C_{9}$ (with $C_{3}$ ), etc. In the general case, the kinetic equations may be written

$$
\begin{aligned}
\mathrm{d}\left[C_{n}\right] / \mathrm{d} t= & \sum_{m=1}^{[n / 2]} k_{m, n-m}\left[C_{m}\right]\left[C_{n-m}\right] \\
& -\sum_{m=1}^{N+1-n} k_{n, m}\left[C_{n}\right]\left[C_{m}\right],
\end{aligned}
$$

where $n$ is the number of carbons in the cluster under study and $N$ is the number of carbons in the largest cluster considered here (i. e., 9). $[n / 2]$ denotes the maximum integer in $n / 2$. The first term (on the RHS) involves the formation of $C_{n}$ via the bimolecular reaction of the smaller clusters $C_{m}$ and $C_{n-m}$ and the second term accounts for the reactions which deplete $C_{n}$ to form larger clusters. Rather than use absolute concentrations of the cluster species, we introduce relative concentrations defined by

$$
\left[C_{n}\right]^{r}=\left[C_{n}\right] / C_{0},
$$

where $C_{0} \equiv \sum_{n=1}^{N} n\left[C_{n}\right]=$ constant. Hence we find

$$
\begin{aligned}
\mathrm{d}\left[C_{n}\right]^{r} / \mathrm{d} t=C_{0} & \left\{\sum_{m=1}^{[n / 2]} k_{m, n-m}\left[C_{m}\right]^{r}\left[C_{n-m}\right]^{r}\right. \\
& \left.-\sum_{m=1}^{N+1-n} k_{n, m}\left[C_{n}\right]^{r}\left[C_{m}\right]^{r}\right\} .
\end{aligned}
$$

This set of coupled equations requires certain assumptions and simplifications. We adopt the approach 

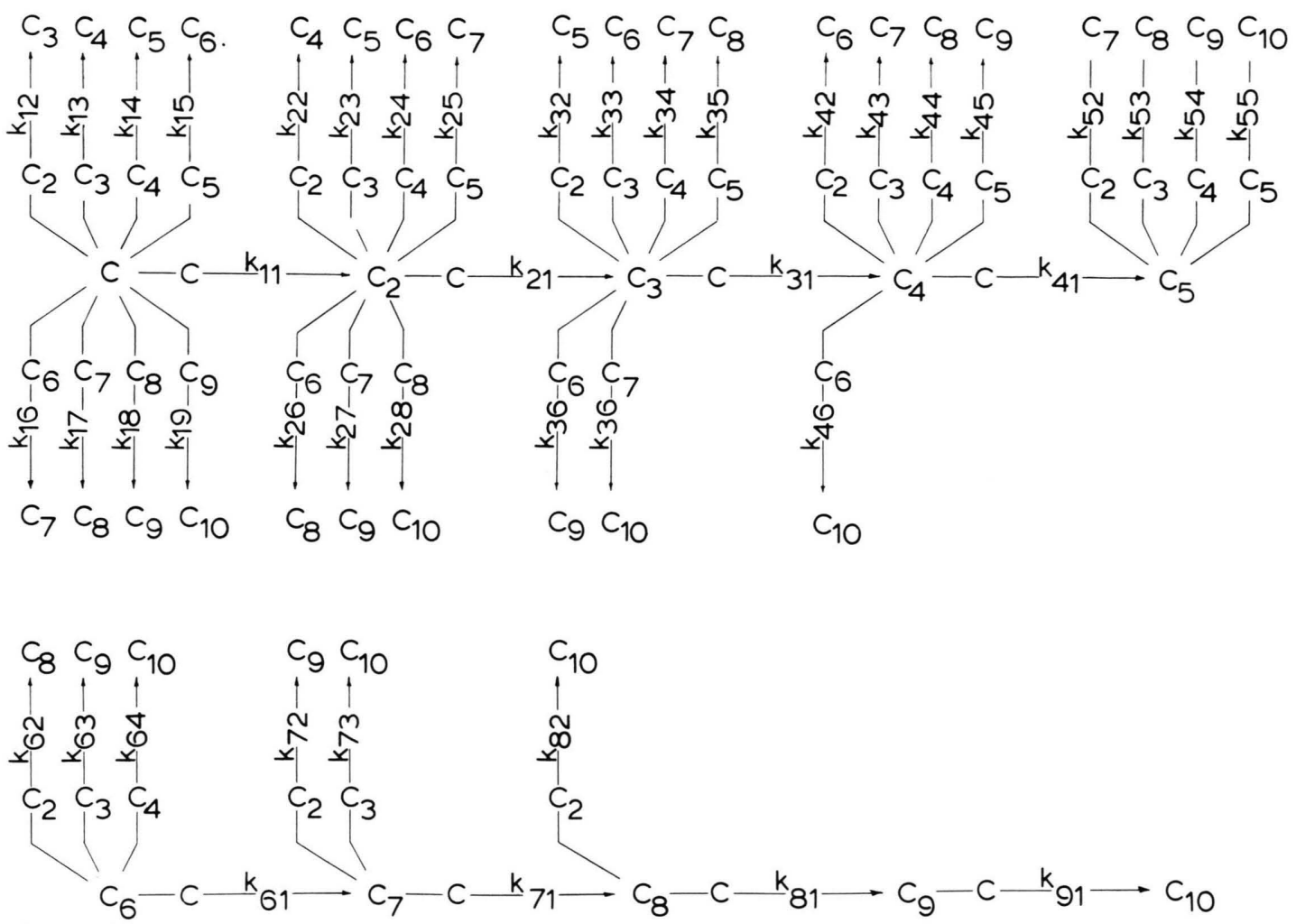

Fig. 5. The net of reactions assumed to be operative between thermally-activated carbon clusters in a rare gas matrix (for $n=1$ to 10$)$.

originally taken by Smoluchowski ${ }^{12}$ and further developed by Waite ${ }^{29}$. In this approach, two species such as $C_{n}$ and $C_{m}$ react upon diffusion in a solid matrix. The kinetic equation for the process is given by

$$
\mathrm{d}\left[C_{n}\right] / \mathrm{d} t=\mathrm{d}\left[C_{m}\right] / \mathrm{d} t=-k_{n, m}\left[C_{n}\right]\left[C_{m}\right],
$$

where

$$
\begin{aligned}
k_{n, m}= & 4 \pi R\left(D_{n}+D_{m}\right) \\
& \cdot\left(1+R\left[\pi\left(D_{n}+D_{m}\right) t\right]^{-1 / 2}\right) .
\end{aligned}
$$

Here $R$ is the radius of the reaction sphere, $D_{n}$ and $D_{m}$ are the diffusion coefficients (for $C_{n}$ and $C_{m}$, respectively) and $t$ is the diffusion time. We assume further that these diffusion coefficients are inversely proportional to the number of carbons in the cluster chain and directly proportional to a Boltzmann-like term involving the diffusional activation barrier, $E_{n}$. Thus, we have

$$
D_{n}=n^{-1 / 2} \mathrm{D}_{0} \exp \left(-E_{n} / R T\right) .
$$

Note the inverse square root dependence of $D_{n}$ on $n$ such that the diffusion rate is inversely proportional to the square root of the molecular weight. This inverse mass dependence reflects our expectation of lower $D_{n}$ diffusion coefficients for higher mass clusters. Although it appears that all (small) cluster sizes can undergo diffusion we make the physically-reasonable assumption that the activation energy for diffusion varies linearly with cluster size. Thus

$$
E_{n}=a+b(n-1),
$$

where $a$ and $b$ are two activation parameters to be fit (vide infra). The influence of factors such as cluster size (volume) and shape on ability to diffuse through a matrix enter indirectly through the activation energy, $E_{n}$.

$D_{0}$ in (5) is estimated in the following manner from the diffusion coefficient for $\mathrm{Cu}$ atoms in solid argon ${ }^{30}$. Solid state theory relates the activation energy, $E$, for autodiffusional processes to temperature by

$$
E \cong 0.032 T_{\mathrm{MP}}, \mathrm{kcal} / \mathrm{mol} \text {, }
$$

where $T_{\mathrm{MP}}$ is the melting point temperature. For $\mathrm{Ar}$, $T_{\mathrm{MP}}=83.8 \mathrm{~K}$, so $E_{\mathrm{Ar}} \cong 2.68 \mathrm{kcal} / \mathrm{mol}$. In this model it is assumed that $E_{\mathrm{Ar}}$ (in $\left.\mathrm{Ar}\right) \cong E_{\mathrm{Cu}}$ (in $\left.\mathrm{Ar}\right) \cong E_{\mathrm{C}}$ (in $\mathrm{Ar}$ ). 
We take the ratio of the $\mathrm{C}$ and $\mathrm{Cu}$ diffusion coefficients as

$$
D_{\mathrm{C}} / D_{\mathrm{Cu}}=\sqrt{M_{\mathrm{Cu}} / M_{\mathrm{C}}}=2.3 \text {, }
$$

where $M_{\mathrm{Cu}}$ is the atomic weight of $\mathrm{Cu}$. Since $D_{\mathrm{Cu}}=4 \times 10^{-17} \mathrm{~cm}^{2} / \mathrm{s}$ at $35 \mathrm{~K}$ in $\mathrm{Ar}^{30} D_{\mathrm{C}}=9.2$ $\times 10^{-17} \mathrm{~cm}^{2} / \mathrm{s}$. Using this value and the above value for $E_{\mathrm{C}}$ in (5) yields $D_{0}$ (in $\mathrm{Ar}$ ) $\cong 4.3 \mathrm{~cm}^{2} / \mathrm{s}$. This value of $D_{0}$ is exactly equal to the average value obtained in two autodiffusional (self-diffusion) experiments for Ar crystals and films using a radioactive tracer-exchange method ${ }^{31}$. In these two experiments the activation energy for self-diffusion was found to be $3.8 \mathrm{kcal} / \mathrm{mol}$.

$C_{0}$ in (2) is obtained by assuming a matrix ratio of $\mathrm{Ar}$ to $\mathrm{C}$ atoms of 200 to 1 , and an $\mathrm{Ar}$ atom radius of $1.9 \AA$. This leads to $C_{0}=1.2 \times 10^{20} \mathrm{C}$ atoms $/ \mathrm{cm}^{3}$. We take the reaction sphere radius $(\mathrm{R})$ as $4 \AA$. In addition to the quantities discussed above, $N$ initial concentrations $\left[C_{N}\right]^{r}(t=0)$ are also required; they are subject to the normalization condition

$$
\sum_{\mathrm{n}} n\left[C_{n}\right]^{r}(t)=1 .
$$

These relative concentrations are estimated initially from available theoretical intensities (transition moments) ${ }^{32}$ and experimental intensity data. They are subsequently varied to effect the fit to experiment.

\section{D) Computational Approach}

Before discussing the computational approach taken, it is useful to briefly review the number and type of parameters in the model. First, there are a total of 25 rate constants in our treatment $\left(k_{1,1-9}, k_{2,2-8}\right.$, $k_{3,3-7}, k_{4,4-6}$, and $k_{5,5}$ ), which are determined by 9 diffusion constants $\left(D_{1}-D_{9}\right)$ and the reaction sphere, $R$ (cf. (4)). Thus, 25 rate constants are replaced by the latter 10 rate parameters. However, our assumption that the diffusion constants can be described with an diffusional activation energy which varies linearly with cluster size, reduces these 10 rate parameters to only 2 , i. e. the $a$ and $b$ in (6). In addition, to solve the coupled equations in (2), we also require initial cluster concentrations. They are firstly chosen from theoretical intensities and experimental intensity data and then varied. In summary, we vary 11 parameters (two activation energy components and 9 concentrations) to effect the fit to 30 observational points ( 5 cluster band intensities each at 6 different temperatures).

To solve the system of coupled differential equations (2), a fourth-order Runge-Kutta algorithm with a fixed

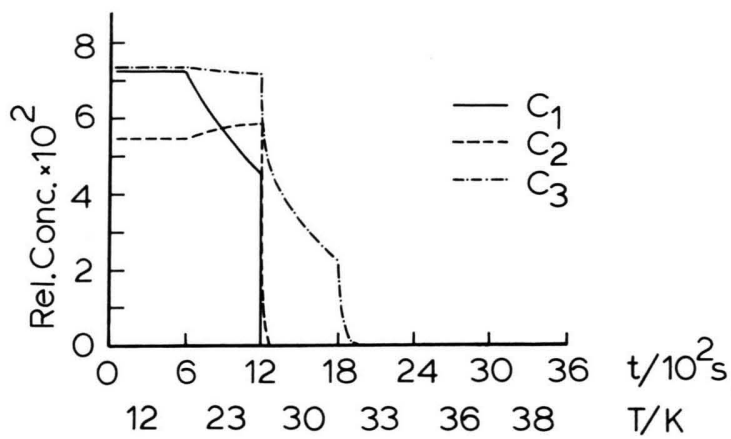

a) Profile for $C_{n}(n=1$ to 3$)$.

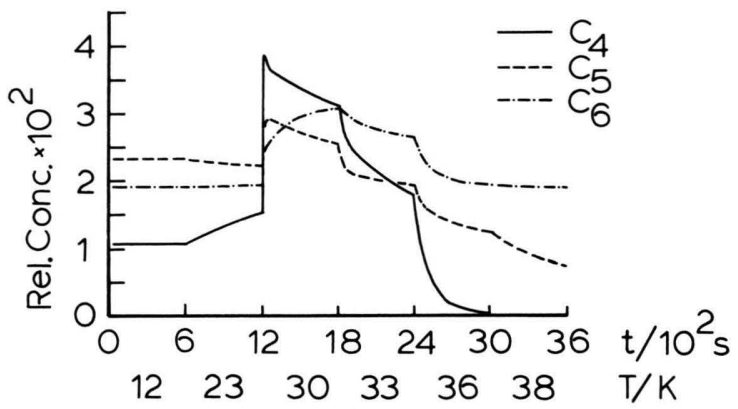

b) Profile for $C_{n}(n=4$ to 6$)$.

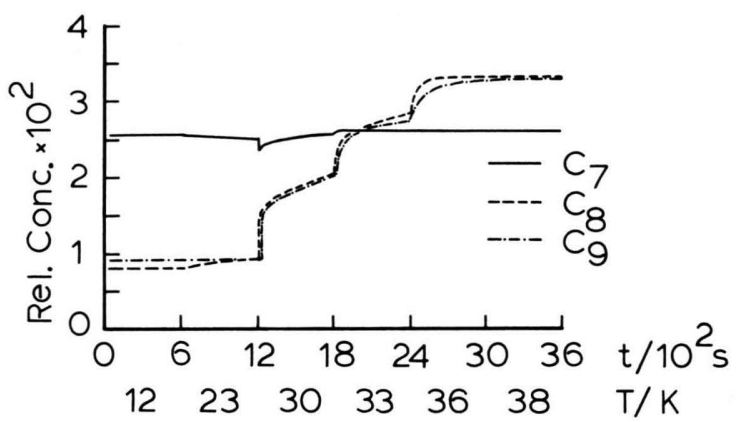

c) Profile for $C_{n}(n=7$ to 9$)$.

Fig. 6. Concentration profiles for the carbon clusters, $C_{n}$ $(1 \leq n \leq 9)$ as function of time (and temperature). The dual abscissa denotes that the matrix is at $12 \mathrm{~K}$ for the time span 0 to $600 \mathrm{~s}$, at $23 \mathrm{~K}$ for the span 600 to $1200 \mathrm{~s}$, etc.

step size was employed ${ }^{33}$. The nine equations $(1 \leq n \leq 9)$ of (2) were integrated as a function of time for six different fixed temperatures $(12,23,30,33,36$, and $38 \mathrm{~K}$ ). For each temperature, integration was carried out for a period of $600 \mathrm{~s}$ (1000 $0.6 \mathrm{~s}$ steps). Concentration ratios, $\left[C_{n}\right]_{\mathrm{T}}^{r} /\left[C_{n}\right]_{12 \mathrm{~K}}^{r}$, were determined because they are directly comparable to experimental intensity ratios, $I_{n}(T) / I_{n}(12 \mathrm{~K})$, since the transition moments cancel. The results of the calculation are shown in Fig- 
Table 1. Initial Concentrations and Activation Energies for Linear Carbon Clusters, $C_{n}$, in Argon Matrices.

\begin{tabular}{lll}
\hline$n$ & $100\left[C_{n}\right]^{r}(t=0)$ & $E_{n}^{*}$ \\
\hline 1 & 7.274 & 1.80 \\
2 & 5.467 & 2.02 \\
3 & 7.345 & 2.24 \\
4 & 1.063 & 2.46 \\
5 & 2.319 & 2.68 \\
6 & 1.914 & 2.90 \\
7 & 2.545 & 3.12 \\
8 & 0.807 & 3.34 \\
9 & 0.906 & 3.56 \\
\hline
\end{tabular}

* $E_{n}=1.8+0.22(n-1) \mathrm{kcal} / \mathrm{mole}$.

ure 6. Two variables, time $(t)$ and temperature $(T)$, are plotted along the abscissa. Their interpretation is as follows: from 0 to $600 \mathrm{~s}$, the concentration profiles $\left(\left[C_{n}\right]_{\mathrm{T}}^{r}\right.$ vs. $\left.t\right)$ are plotted for $12 \mathrm{~K}$. Note that no change in $\left[C_{n}\right]^{r}$ is predicted for any species. At $t=600 \mathrm{~s}, T$ was raised "instantaneously" to $23 \mathrm{~K}$ and in the interval 600-1200 s the time evolution of $\left[C_{n}\right]^{r}$ plotted. In this time-temperature interval $\left[C_{1}\right]^{r}$ falls by $\sim 50 \%,\left[C_{2}\right]^{r}$ rises, while $\left[C_{3,5,6}\right]^{r}$ all decline slightly. At $1200 \mathrm{~s}$ the temperature is raised to $30 \mathrm{~K}$ where significant changes occur. $\left[C_{1,2}\right]^{\mathrm{r}}$ drop precipitously to practically zero, $\left[C_{3}\right]^{r}$ declines strongly, while all the rest jump in concentration, $\left[C_{4}\right]^{r}$ by more than $300 \%$. Similar advances in temperature to a maximum of $38 \mathrm{~K}$ influence the species differently and may be followed readily on the plot.

To obtain the theoretical points in Fig. 4, the relative concentrations, $\left[C_{n}\right]^{r}$, were taken at the end of each $600 \mathrm{~s}$ interval. This plot corresponds more closely to experiment since no time dependence is included; only the static concentrations (IR intensities) of the species after annealing at a particular temperature are recorded. Note that the curves have all been normalized to the concentrations/intensities at $12 \mathrm{~K}$; thus all growth/decay curves start at an ordinate of 1.0. The fit to the known clusters is reasonably good. The optimized initial concentrations and activation energies found are presented in Table 1. Comparison of calculated and experimental intensity (relative concentration) ratios (i.e., $I_{n}(T) / I_{n}(12 \mathrm{~K})$ ) is given in Table 2. While some discrepancies are evident, the overall annealing trends mimic the experimental data rather well. The $C_{3}$ and $C_{5}$ species decay with increasing temperature with $C_{3}$ commencing at a lower temperature. The $C_{6}$ cluster grows to a maximum at $\sim 33 \mathrm{~K}$ and declines thereafter, while $C_{9}$ climbs $400 \%$ up to $\sim 36 \mathrm{~K}$ at which temperature it starts to level off. The unknown clusters are each predicted to behave differently. The $C_{7}$ chain is predicted to show little variation with temperature. On the other hand, $C_{4}$ is predicted to increase dramatically at $30 \mathrm{~K}$ and then drop off. $C_{8}$ is expected to increase quickly at $30 \mathrm{~K}$ and then gently increase with further temperature increases. Of the prominent unassigned IR bands the ones at $2128 \mathrm{~cm}^{-1}$ and $1894 \mathrm{~cm}^{-1}$ are observed to vary little with annealing temperature increase. We therefore assign them to the linear $C_{7}$ species.

\section{Discussion}

In this paper evidence has been presented on the aggregation of small carbon clusters upon annealing of the argon matrix in which they are trapped. It has been observed that the $C_{3}$ and $C_{5}$ clusters decay when the matrix is warmed while the $C_{6}$ and $C_{9}$ species grow. A kinetic reaction network which includes solid state diffusion of all possible pairwise combinations of small clusters $(1 \leq n \leq 10)$ has been shown to account reasonably well for the growth/decay patterns of the known clusters. Activation energies for these aggregation steps have been extracted under the assumption that they increase linearly with cluster size. They all fall in the range $1.8 \mathrm{kcal} / \mathrm{mol}(n=1)$ to $3.6 \mathrm{kcal} / \mathrm{mol}$ $(n=9)$. The fit to the growth/decay curves with temperature (cf. Fig. 4) mimics the experimental points rather well, with the exception of $C_{3}$ : its fit falls off much faster with temperature than observed. This may result from a too-small activation energy. The calculational results are very sensitive to the activation energies chosen. If the assumed linear form for the activation energy is not applicable to the $C_{3}$, this could result in a erroneously small energy barrier and a reaction which progresses too rapidly.

In addition to the fit to the known clusters, the results also yield predicted profiles for the unassigned clusters (i. e., those clusters whose vibrational frequencies are forbidden $\left(C_{2}\right)$ or, as yet, not conclusively determined $\left.\left(C_{7}, C_{8}\right)\right)$. The predictions are given in Table 3 (and for $C_{7}$ in Table 2 also). Interestingly, the prediction for $C_{8}$ closely parallels that for $C_{9}$ (cf. Figure 6).

The most significant prediction which emerges from the calculations concerns the $C_{7}$ clusters. It may be seen in Fig. 6 that its concentration is predicted to vary negligibly over the $12-38 \mathrm{~K}$ temperature range. 
Table 2. Experimental and Theoretical (in parentheses) Intensity Ratios, $I_{n}(T) / I_{n}(12 \mathrm{~K})$, for Various Carbon Clusters ${ }^{\mathrm{a}}$.

\begin{tabular}{lllllll}
\hline$T / \mathrm{K}$ & $t / 10^{2} \mathrm{~s}$ & $\begin{array}{l}C_{3} \\
2039 \mathrm{~cm}^{-1}\end{array}$ & $\begin{array}{l}C_{5} \\
2164 \mathrm{~cm}^{-1}\end{array}$ & $\begin{array}{l}C_{6} \\
1952 \mathrm{~cm}^{-1}\end{array}$ & $\begin{array}{l}C_{7} \\
2128 \mathrm{~cm}^{-1}\end{array}$ & $\begin{array}{c}C_{9} \\
1998 \mathrm{~cm}^{-1}\end{array}$ \\
\hline 12 & $0-6$ & $1.00(1.00)$ & $1.00(1.00)$ & $1.00(1.00)$ & $1.00(1.00)$ & $1.00(1.00)$ \\
23 & $6-12$ & $0.95(0.98)$ & $1.01(0.97)$ & $1.02(1.01)$ & $1.06(0.98)$ & $1.22(1.16)$ \\
30 & $12-18$ & $0.79(0.31)$ & $0.82(1.10)$ & $1.11(1.62)$ & $1.12(1.01)$ & $2.57(2.52)$ \\
33 & $18-24$ & $0.52(0.00)$ & $0.56(0.84)$ & $1.18(1.40)$ & $1.12(1.03)$ & $4.05(3.52)$ \\
36 & $24-30$ & $0.27(0.00)$ & $0.44(0.54)$ & $1.12(1.02)$ & $1.12(1.03)$ & $4.00(4.13)$ \\
38 & $30-36$ & $0.14(0.00)$ & $0.31(0.32)$ & $0.94(1.01)$ & $1.06(1.03)$ & $4.00(4.13)$ \\
\hline
\end{tabular}

a Experimental data for the clusters $C_{3}, C_{5}, C_{6}, C_{7}$, and $C_{9}$ were used in the fit (see text). RMS Error $=0.24$.

Table 3. Theoretical Intensity Ratios, $I_{n}(T) / I_{n}(12 \mathrm{~K})$ for $C_{1}$, $C_{2}, C_{4}, C_{7}$, and $C_{8}{ }^{\text {. }}$.

\begin{tabular}{lclllll}
\hline$T / \mathrm{K}$ & $t / 10^{2} \mathrm{~s}$ & $C_{1}$ & $C_{2}$ & $C_{4}$ & $C_{7}$ & $C_{8}$ \\
\hline 12 & $0-6$ & 1.00 & 1.00 & 1.00 & 1.00 & 1.00 \\
23 & $6-12$ & 0.63 & 1.08 & 1.45 & 1.06 & 1.00 \\
30 & $12-18$ & 0.00 & 0.00 & 2.95 & 1.12 & 2.23 \\
33 & $18-24$ & 0.00 & 0.00 & 1.69 & 1.12 & 3.03 \\
36 & $24-30$ & 0.00 & 0.00 & 0.04 & 1.12 & 3.62 \\
38 & $30-36$ & 0.00 & 0.00 & 0.00 & 1.06 & 3.64 \\
\hline
\end{tabular}

a RMS error $=0.24$ for fit to $C_{3}, C_{5}, C_{6}, C_{7}$, and $C_{8}$; note that $I_{n}(T) / I_{n}(12 \mathrm{~K})=\left[C_{n}\right]_{(T)}^{r} /\left[C_{n}\right]_{(12 \mathrm{~K})}^{r}=\left[C_{n}\right]_{(T)} /\left[C_{n}\right]_{(12 \mathrm{~K})}$.

Of the major bands in the $\mathrm{C}-\mathrm{C}$ stretch region, only the $2128 \mathrm{~cm}^{-1}$ and $1894 \mathrm{~cm}^{-1}$ bands are observed to display this independence of temperature. On the basis of this similarity we assign these two bands to the

[1] For a recent review, see W. Weltner, Jr. and R. Van Zee, Chem. Rev. 89, 1713 (1989).

[2] K. R. Thompson, R. L. DeKock, and W. Weltner, Jr., J. Amer. Chem. Soc. 93, 4688 (1971).

[3] W. Krätschmer and K. Nachtigall, in Polycyclic Aromatic Hydrocarbons and Astrophysics, eds. A. Léger, L. d'Hendecourt and N. Boccara, D. Reidel Publishing Co., Dordrecht 1987.

[4] M. Vala, T. M. Chandrasekhar, J. Szczepanski, and R. Pellow, in Materials Chemistry at High Temperature, Vol. II, ed. J. Hastie, Humana Press, 1990; and High Temperature Science 27, 19 (1990).

[5] J. Kurtz and D. Huffman, J. Chem. Phys. 92, 30 (1990).

[6] I. Yamada, H. Usui, and T. Takagi, in Metal Clusters, Eds. F. Träger and G. zu Putlitz, Springer-Verlag, Berlin 1986, p. $37 \mathrm{ff}$.

[7] A. W. Castleman, Jr. and R. G. Keesee, ibid, p. $67 \mathrm{ff}$.

[8] a) J. Bernholc and J. C. Phillips, J. Chem. Phys., 85(6), 3258 (1986); b) J. Bernholc and J. C. Phillips, Phys. Rev. B, 33, 7395 (1986).

[9] G. A. Ozin and H. Huber, Inorg. Chem. 17, 155 (1978).

[10] M. Moskovits and J. E. Hulse, J. Chem. Soc. Faraday Trans. 2 73, 471 (1977).

[11] T. Nishiya, N. Hirota, H. Shinohara, and N. Nishi, J. Phys. Chem. 89, 2260 (1985). linear $C_{7}$ cluster. As mentioned in Sect. IIIF, Saykally and co-workers ${ }^{24}$ have recently assigned a gas phase band at $2138 \mathrm{~cm}^{-1}$ to linear $\mathrm{C}_{7}$. The $10 \mathrm{~cm}^{-1}$ gas-tomatrix shift for the $2128 \mathrm{~cm}^{-1}$ band is larger than the $5 \mathrm{~cm}^{-1}$ gas-to-matrix seen for $C_{5}$, but is still only a $0.5 \%$ shift, a not unreasonable value ${ }^{34}$. Finally, Martin, Francois and Gijbels ${ }^{23}$ have argued persuasively on theoretical grounds that the $2128 \mathrm{~cm}^{-1}$ band should be assigned to linear $C_{7}$.

\section{Acknowledgements}

We gratefully acknowledge the National Science Foundation for its support of this research under Grant No. CHE-8903133. We also thank Dr. J. M. L. Martin for a preprint of his work before publication.

[12] a) M. V. Smoluchowski, Z. Phys. Chem. 92, 192 (1917); b) cf. S. Chandrasekhar, Rev. Mod. Phys. 15, 1 (1943).

[13] J. Szczepanski and M. Vala, J. Phys. Chem. 95, 2792 (1991).

[14] R. J. Van Zee, R. F. Ferrante, K. J. Zeringue, and W. Weltner, Jr., J. Chem. Phys. 88, 3465 (1988).

[15] W. Weltner, Jr., P. N. Walsh, and C. L. Angell, J. Chem. Phys. 40, 1299 (1964).

[16] W. Weltner, Jr. and D. McLeod, Jr., J. Chem. Phys. 40, 1305 (1964).

[17] L. N. Shen and W. R. M. Graham, J. Chem. Phys. 91, 5115 (1989).

[18] M. Vala, T. M. Chandrasekhar, J. Szczepanski, Richard Van Zee, and W. Weltner, Jr., J. Chem. Phys. 90, 595 (1989).

[19] R. Pellow and M. Vala, Z. Physik D 15, 171 (1990).

[20] a) N. Moazzen-Ahmadi, A. R. W. McKellar, and T. Amano, Chem. Phys. Lett. 157, 1 (1989); b) J. R. Heath, A. L. Cooksey, M. H. W. Gruebele, C. A. Schuttenmaer, and R. J. Saykally, Science 244, 564 (1989).

[21] P. F. Bernath, K. H. Hinkle, and J. J. Keady, Science 244, 562 (1989).

[22] M. Vala, T. M. Chandrasekhar, J. Szczepanski, and R. Pellow, J. Mol. Structure 222, 209 (1990). 
[23] J. M. L. Martin, J. P. Francois, and R. Gijbels, J. Chem. Phys. 93, 8850 (1990).

[24] J. R. Heath, R. A. Sheeks, A. L. Cooksy, and R. J. Saykally, Science 249, 895 (1990).

[25] a) J. R. Heath and R. J. Saykally, J. Chem. Phys. 93, 8392 (1991); b) J. R. Heath and R. J. Saykally, J. Chem. Phys. 94, 1724 (1991).

[26] J. Szczepanski, R. Pellow, and M. Vala, in preparation.

[27] K. S. Pitzer and E. Clementi, J. Amer. Chem. Soc. 81, 4477 (1959).

[28] W. R. M. Graham, K. I. Dismuke, and W. Weltner, Jr. Astrophys. J. 204, 301 (1976).

[29] T. R. Waite, Phys. Rev. 107 (2), 463 (1957).
[30] M. Moskovits and G. A. Ozin in Cryochemistry, eds. M. Moskovits and G. A. Ozin, Wiley-Intersci., New York 1976, p. 335.

[31] A. V. Chadwick, in Mass Transport in Solids, eds. F. Bénière and C. R. A. Catlow, NATO ASI Series B: Physics, 97, 309 (1981).

[32] J. Kurtz and L. Adamowicz, Astrophys. J. 370, 784 (1991).

[33] W. H. Press, B. P. Flannery, S. A. Teukolsky, and W. T. Vetterling, Numerical Recipes, Cambridge University Press, London 1989, p. 550.

[34] M. E. Jacox, Rev. Chem. Intermed. 2, (1) 1-36 (1978). 